\title{
On a covering problem for equilateral triangles
}

\author{
Adrian Dumitrescu * \\ Department of Computer Science \\ University of Wisconsin-Milwaukee \\ Milwaukee, WI 53201-0784, USA \\ Email: ad@cs . uwm . edu
}

\author{
Minghui Jiang ${ }^{\dagger}$
}

Submitted: July 22, 2006; Accepted: Feb 26, 2008; Published: Feb 29, 2008

Mathematics Subject Classification: 52C15

\begin{abstract}
Let $T$ be a unit equilateral triangle, and $T_{1}, \ldots, T_{n}$ be $n$ equilateral triangles that cover $T$ and satisfy the following two conditions: (i) $T_{i}$ has side length $t_{i}\left(0<t_{i}<1\right)$; (ii) $T_{i}$ is placed with each side parallel to a side of $T$. We prove a conjecture of Zhang and Fan asserting that any covering that meets the above two conditions (i) and (ii) satisfies $\sum_{i=1}^{n} t_{i} \geq 2$. We also show that this bound cannot be improved.
\end{abstract}

\section{Introduction}

Inspired by an old problem of Erdős about packing smaller squares in a unit square [2, 3, 4], Zhang and Fan [7] have recently considered the following covering problem for the equilateral triangle. Let $T$ be a unit equilateral triangle, and $\mathcal{T}=\left\{T_{1}, \ldots, T_{n}\right\}$ be a set of $n$ equilateral triangles that cover $T$ and satisfy the following two conditions: (i) $T_{i}$ has side length $t_{i}(0<$ $t_{i}<1$ ); (ii) $T_{i}$ is placed with each side parallel to a side of $T$. All triangles are viewed as closed sets. An example of such a covering with 5 triangles is shown in Fig. 1(a).

Define

$$
U(n)=\inf _{\substack{\mathcal{T}: \text { covering } \\|\mathcal{T}|=n}} \sum_{i=1}^{n} t_{i} .
$$

Since the triangles in the covering are smaller than $T$, each triangle $T_{i}$ can cover at most one vertex of $T$, so the condition $n \geq 3$ is necessary. Recently Zhang and Fan showed the following upper bounds on $U(n): U(n) \leq 3-\frac{4}{n}$ for even $n \geq 4 ; U(n) \leq 4-\frac{6}{n-3}$ for odd

${ }^{*}$ Supported in part by NSF CAREER grant CCF-0444188.

${ }^{\dagger}$ Supported in part by NSF grant DBI-0743670. 
$n \geq 7$. In particular $U(4) \leq 2$ follows. They also found that $U(3) \leq 2$, and $U(5)<9 / 4$. It should be noted here that the inequality $U(k) \leq 2$ for some $k \geq 3$ does not imply for instance $U(k+1) \leq 2$, so in particular having $U(3) \leq 2$ does not imply a similar bound for larger $n$; this is so because the values $U(n)$ are defined for each specific value of $n$.

For the opposite direction, Zhang and Fan conjectured that $U(n) \geq 2$ holds for each $n \geq 3$. Here we prove that $\sum_{i=1}^{n} t_{i} \geq 2$ holds for any covering that meets conditions (i) and (ii) above; therefore $U(n) \geq 2$ for each $n \geq 3$. We also improve on all the above-mentioned upper bounds (for $n \geq 5$ ) and thus obtain exact bounds on $U(n)$ for every $n \geq 3$ :

Theorem 1 For any covering of $T$ with $n \geq 3$ triangles, which satisfies conditions (i) and (ii), we have $\sum_{i=1}^{n} t_{i} \geq 2$. This bound is best possible, i.e., for each $n \geq 3$, we have $U(n)=2$.

We can similarly define coverings of the unit square with homothetic smaller squares and ask the same question. It turns out that the answer is the same, but the proof is much simpler; we give the details in the last section.

Definitions. A translate of a set $X \subset \mathbb{R}^{d}$ is a set $X+t$, with $t \in \mathbb{R}^{d}$. A (positive) homothetic copy is a scaled translate $\lambda X+t$, for $\lambda>0$. A negative homothetic copy is a scaled translate $\lambda X+t$, for $\lambda<0$.

\section{Proof of Theorem 1}

Let $n \geq 3$ be fixed. We start with the proof of the lower bound: in any covering satisfying conditions (i) and (ii), $\sum_{i=1}^{n} t_{i} \geq 2$ holds. Two triangles $\Delta_{1}$ and $\Delta_{2}$ are said to be overlapping (or intersecting) if they have at least one common point. Let $\mathcal{T}=\left\{T_{1}, \ldots, T_{n}\right\}$ be $n$ equilateral triangles that cover $T=\triangle A B C$. An equilateral triangle $T_{A} \subset T$ is called a special triangle corresponding to vertex $A$ if $T_{A}$ is a smaller homothetic copy of $T$ having $A$ as a common vertex, e.g., $\triangle A U V$ in Fig. 1(a). Special triangles $T_{B}$ and $T_{C}$, corresponding to the other two vertices $B$ and $C$, are defined similarly.

We have two types of equilateral triangles in the covering set: (1) a positive copy is a triangle homothetic to $T$, for example $\triangle E F G$ in Fig. 1(a); (2) a negative copy is a triangle homothetic to a reflection of $T$ about the side $B C$, for example $\triangle M N P$ in Fig. 1(a). With our prior definitions, these are positive and negative homothetic copies with $-1<\lambda<1$. See also [1,5] for references to other covering problems using positive and negative copies.

We first give a short outline of the proof: In a finite number of operations we transform $\mathcal{T}$ into three equilateral special triangles $T_{A}, T_{B}, T_{C}$ of side lengths $x, y, z<1$ which cover $T$, so that $T_{A}$ covers $A, T_{B}$ covers $B$, and $T_{C}$ covers $C$, and $T_{A}, T_{B}, T_{C} \subset T$, so that $x+y+z \leq$ $\sum_{i=1}^{n} t_{i}$. We then easily derive that $x+y+z \geq 2$ must hold, which completes the proof of the lower bound. The transformation procedure maintains the following three invariants after each operation:

1. The current set of triangles $\mathcal{T}$ forms a covering set (i.e., $\mathcal{T}$ covers $T$ ) that includes three special triangles.

2. The sum of the side lengths of all triangles in the current covering set is non-increasing. 


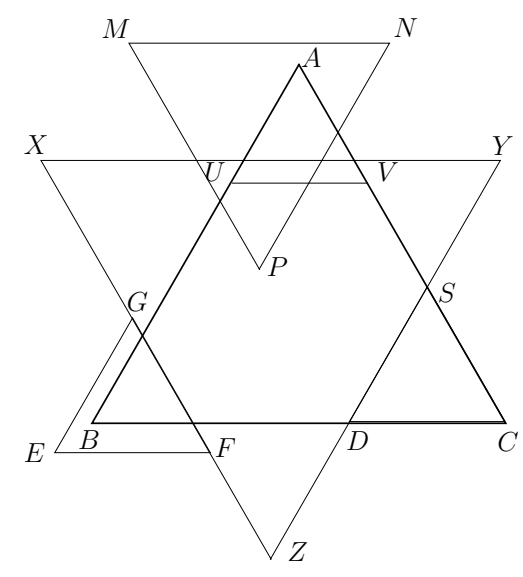

(a)

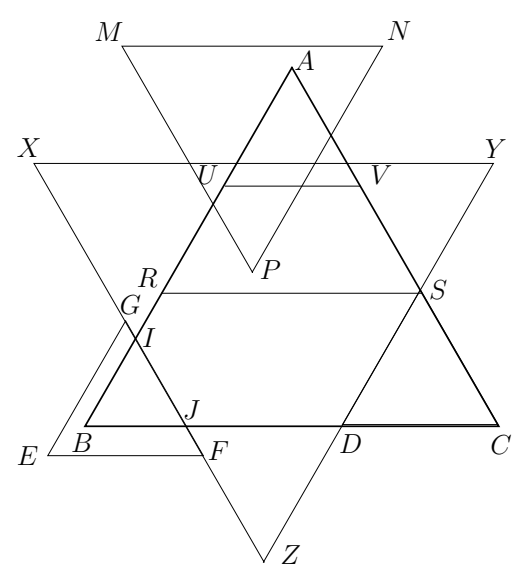

(b)

Figure 1: (a) Covering of $T=\triangle A B C$ with 5 triangles: $\triangle M N P, \triangle A U V, \triangle E F G, \triangle C D S$, and $\triangle X Y Z$. (b) Obtaining the three special triangles in phase 1: $T_{A}=\triangle A R S, T_{B}=\Delta B I J$, and $T_{C}=$ $\triangle C D S$.

3. The side length of each triangle in the current covering set is strictly less than 1 (and strictly positive).

The transformation procedure has two phases.

Phase 1. Refer to Fig. 1(b). We first replace all positive triangles by clipping them to $T$ : $T_{i} \rightarrow T_{i} \cap T$. Next, for each vertex $A, B$, and $C$, replace all triangles (positive or negative) that cover that vertex by a special triangle corresponding to that vertex; the side length of the special triangle is the maximum side length of the replaced triangles. Note that the special triangle constructed still covers the part of $T$ initially covered by the replaced triangles, and that its side length is at most the sum of the side lengths of the replaced triangles. For instance, in phase 1 for vertex $A$, the two triangles $\triangle M N P$ and $\triangle A U V$ covering $A$ are replaced by the special triangle $\triangle A R S$. For vertex $B$, the triangle $\triangle G E F$ which contains $B$ is replaced by the smaller triangle $\triangle B I J$. Phase 1 leads to three special triangles, one for each vertex: $\triangle A R S, \Delta B I J$, and $\triangle C D S$ for the example in Fig. 1(a). Note that after phase 1, each positive triangle in the covering set which is not special has at most one side overlapping with a side of $T$.

Phase 2. Refer to Fig. 2 and Fig. 3. Phase 2 consists of several extend/shrink operations that modify the current covering set of triangles. An extend/shrink operation takes as input a special triangle and a triangle $T^{\prime}$ in the current covering set (possibly not from the original covering set) that overlaps with it. If the three current special triangles cover $T$, the transformation procedure ends and we go to the last step in the proof. Otherwise (the three special triangles do not cover $T$ ), since $\mathcal{T}$ covers $T$ by the first invariant, we have $|\mathcal{T}| \geq 4$, and for each $\gamma \in\{A, B, C\}, T_{\gamma}$ overlaps with some triangle $T^{\prime} \in \mathcal{T} \backslash\left\{T_{A}, T_{B}, T_{C}\right\}$. In our procedure, we choose $\gamma \in\{A, B, C\}$ for which the side length is the minimum among the special triangles. We then apply the extend/shrink operation conforming to the case analysis described below. In the operation, one special triangle (not necessarily $T_{\gamma}$ ) is extended so that the resulting side length is strictly less 
than 1 , while the other triangle $T^{\prime}$ shrinks (or disappears completely, being merged in the special triangle). We now follow with the technical details.

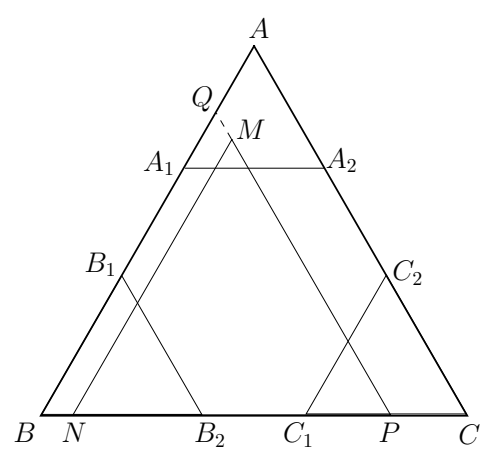

(a)

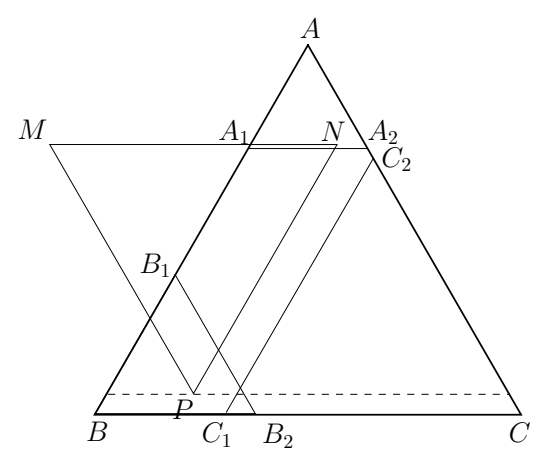

(b)

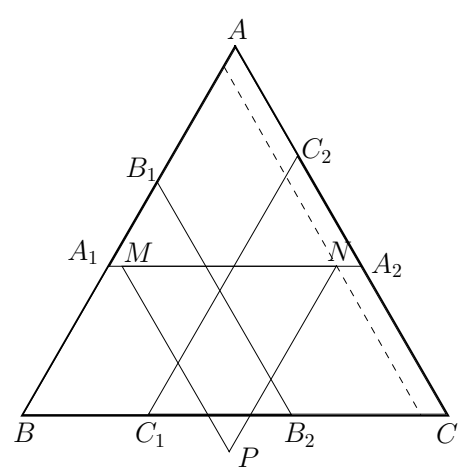

(c)

Figure 2: (a) Extend/shrink operation on $T_{B}=\triangle B B_{1} B_{2}$ and a positive triangle $T^{\prime}=\triangle M N P$ (phase 2, case 1) resulting in the new special triangle $T_{B}=\triangle B P Q$ which replaces the two. (b) Extend/shrink operation on $T_{A}=\triangle A A_{1} A_{2}$ and a negative triangle $T^{\prime}=\Delta M N P$ (phase 2, case 2a). (c) Extend/shrink operation on $T_{B}=\Delta B B_{1} B_{2}$ and a negative triangle $T^{\prime}=\Delta M N P$ (phase 2, case 2b).

Let $\mathcal{T}$ denote the set of triangles covering $T$ in the current step, and $\mathcal{T}^{\prime} \subset \mathcal{T} \backslash\left\{T_{A}, T_{B}, T_{C}\right\}$ be the set of triangles overlapping at least one of the three special triangles $T_{A}=\triangle A A_{1} A_{2}$, $T_{B}=\Delta B B_{1} B_{2}$, and $T_{C}=\Delta C C_{1} C_{2}$. Without loss of generality we assume that $T_{A}$ has the minimum side length among the special triangles, i.e., $\gamma=A$. So we have $T_{A}$ intersecting with $T^{\prime} \in \mathcal{T}^{\prime}$. Case 1 (resp. 2) corresponds to $T^{\prime}$ being a positive (resp. negative) triangle.

Case 1: $T^{\prime}$ is a positive triangle; see Fig. 2(a). If its horizontal side $N P$ does not overlap with $B C$ (i.e., $N P$ is above $B C$ ), $T_{A}$ is extended until $A_{1} A_{2}$ passes through $N($ and $P$ ) and $T_{A}$ contains $T^{\prime}$. Otherwise, since $T_{A}$ has the minimum side length, $T^{\prime}$ must intersect both $T_{B}$ and $T_{C}$. Triangle $T_{B}$ is extended until $B_{1} B_{2}$ passes through $P$ (and $M$ ) and $T_{B}$ contains $T^{\prime}$. In either situation, $T^{\prime}$ is removed from the current covering set after the operation.

Case 2a: $T^{\prime}=\triangle M N P$ is a negative triangle with vertex $P$ lying above $B C$, as in Fig. 2(b). Then $T_{A}$ is extended until $A_{1} A_{2}$ passes through $P$ and $T_{A}$ contains $T^{\prime}$. Then $T^{\prime}$ is removed from the current covering set.

Case $2 b: T^{\prime}=\triangle M N P$ is a negative triangle with vertex $P$ lying below $B C$, so that $B C$ is the only side of $T$ intersected by $T^{\prime}$, as in Fig. 2(c). Since $T_{A}$ has the minimum side length, $T^{\prime}$ must overlap with both $T_{B}$ and $T_{C}$. $T_{B}$ is extended until $B_{1} B_{2}$ passes through $N$ and $T_{B}$ contains $T^{\prime}$. Then $T^{\prime}$ is removed from the current covering set.

Case 2c: $T^{\prime}=\triangle M N P$ is a negative triangle with vertex $P$ lying below $B C$, so that $T^{\prime}$ intersects two or all three sides of $T$, as in Fig. 3(a) and Fig. 3(b). $T_{A}$ is extended until $A_{1} A_{2}$ lies below the lowest point(s) of intersection of $T^{\prime}$ with $A B$ and $A C$. Such a position (the choice is not unique) is the dashed line in the figure; for example, $A_{1} A_{2}$ can be chosen very close to, and above $B C . T^{\prime}$ shrinks correspondingly so that its horizontal side is along the same dashed line. Observe that the resulting shrunk triangle intersects now only one side of $T$, namely $B C$. 


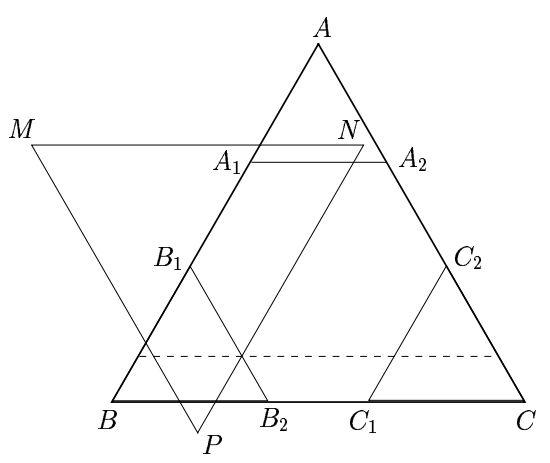

(a)

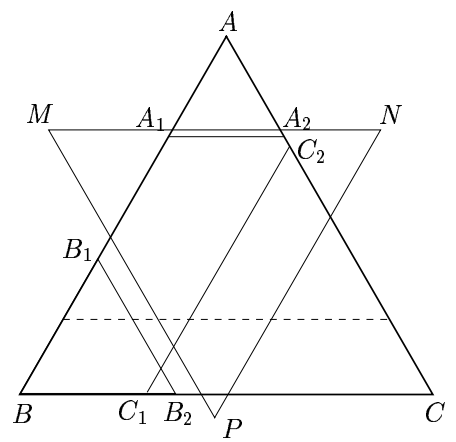

(b)

Figure 3: (a) and (b): Extend/shrink operations on $T_{A}=\Delta A A_{1} A_{2}$ and a negative triangle $T^{\prime}=$ $\triangle M N P$ (phase 2, case 2c).

Note that any of the original triangles $T_{i}$ in the covering set may participate in at most two extend/shrink operations after which it disappears from the covering set. In all these cases, the two side lengths change as follows (after an operation): either as $(x, t) \rightarrow\left(x+t^{\prime}, 0\right)$, where $t^{\prime} \leq t$ and $x+t^{\prime}<1$; or as $(x, t) \rightarrow\left(x+t^{\prime}, t-t^{\prime \prime}\right)$, where $t^{\prime} \leq t^{\prime \prime}$ and $t-t^{\prime \prime}>0$ and $x+t^{\prime}<1$. That is, the side length increase for the special triangle does not exceed the side length reduction for the shrunk (or eliminated) triangle, therefore the sum of the side lengths of all triangles in the covering does not increase. The resulting triangle(s) always cover the part of $T$ which was covered by the replaced triangles prior to the extend/shrink operation: this property follows easily from the geometry of equilateral triangles, using the fact that all the triangles in the covering have their sides parallel to the sides of $T$. The net effect of our procedure is that all triangles that are not special are finally eliminated through extend/shrink (or merge) operations.

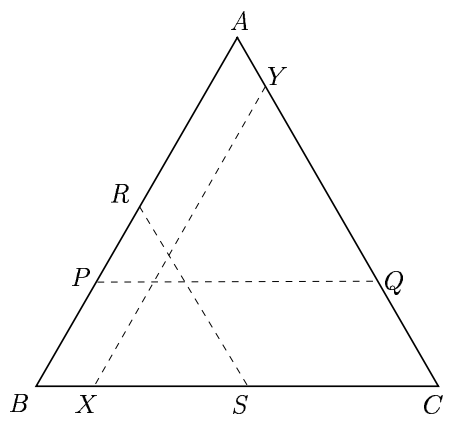

(a)

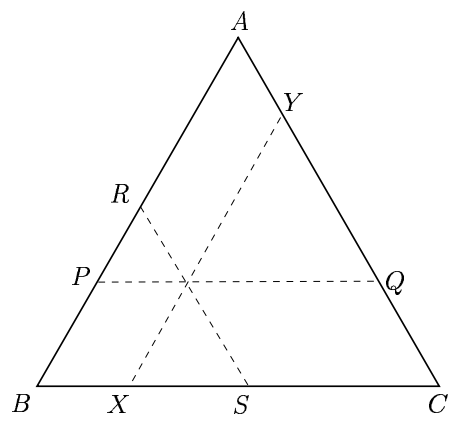

(b)

Figure 4: Two coverings of $\triangle A B C$ with 3 triangles: $\triangle A P Q, \triangle B R S$, and $\triangle C X Y$.

At the end of phase 2, after not more than $2 n$ extend/shrink operations, the covering set consists of three special triangles with side lengths $x, y, z<1$ which cover $T$, as in Fig. 4. It remains to prove that $x+y+z \geq 2$. Put $x=|A P|=|A Q|=|P Q|, y=|B R|=|B S|=|R S|$, and $z=|C X|=|C Y|=|X Y|$. If $x$ and $y$ are fixed, and $P Q, R S, X Y$ are not concurrent, 
$x+y+z$ could be further reduced by moving $X Y$ parallel to itself and closer to $C$ (while maintaining the covering property) until the three segments are concurrent. Then

$$
x+y+z=|A P|+|B R|+|C Y|=1+|P R|+|C Y|=1+|A Y|+|Y C|=1+1=2,
$$

and the lower bound follows.

It is not difficult to construct a covering ${ }^{1}$ showing that $U(n) \leq 2$, for each $n \geq 3$, see Fig. 5 . Let $\varepsilon>0$ be arbitrary small, and set $\delta=\varepsilon$ for $n=3$, and $\delta=\frac{\varepsilon}{n-3}$ for $n \geq 4$. We cover $T$ by a large triangle $\triangle A R S$ of side length $x$, and by $n-1$ partially overlapping congruent small triangles $\ldots, \Delta U V W, \ldots$ of side length $y$, where

$$
x=|A R|=1-\delta ; \quad y=|V W|=\frac{x}{n-1}+\delta=\frac{1+(n-2) \delta}{n-1} .
$$

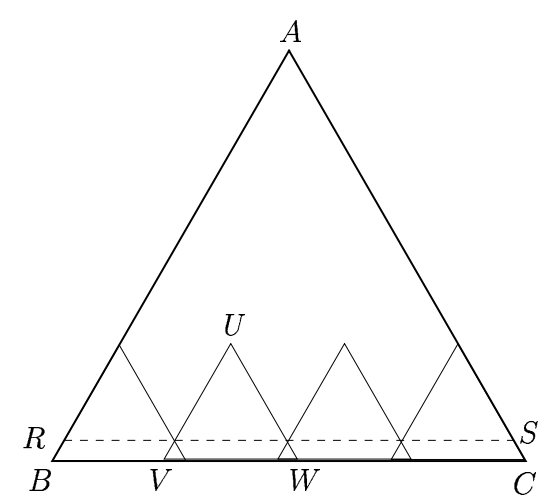

Figure 5: A covering of $T=\Delta A B C$ with $n=5$ triangles, where $\sum_{i=1}^{n} t_{i} \leq 2+\varepsilon$.

We have a covering with

$$
\sum_{i=1}^{n} t_{i}=x+(n-1) y=2+(n-3) \delta \leq 2+\varepsilon .
$$

Making $\varepsilon$ arbitrary small yields $U(n) \leq 2$, as claimed, and the proof of Theorem 1 is complete.

\section{Covering the square with smaller squares}

Let $Q$ be a unit square and $\mathcal{Q}=\left\{Q_{1}, \ldots, Q_{n}\right\}$ be a set of $n$ homothetic smaller squares with side lengths $0<t_{i}<1$ which cover $Q$. Since the squares in the covering are smaller than $Q$, each square $Q_{i}$ can cover at most one vertex of $Q$, so the condition $n \geq 4$ is necessary. For a given $n \geq 4$, define similarly $V(n)=\inf _{\mathcal{Q}} \sum_{i=1}^{n} t_{i}$, where $t_{i}$ denotes the side length of the $i$ th

\footnotetext{
${ }^{1}$ The coverings from [7] mentioned in the introduction accounting for various upper bounds on $U(n)$, as well as ours, are also minimal in a certain sense, as defined in [7].
} 
square $Q_{i}$, and the infimum is taken over all coverings $\mathcal{Q}$ with $n$ homothetic smaller squares $\left(0<t_{i}<1\right)$.

As in our previous proof for triangles, we show that $\sum_{i=1}^{n} t_{i} \geq 2$ holds for any covering with $n$ homothetic smaller squares with side lengths $0<t_{i}<1$. Notice that any square in the covering cannot simultaneously cover parts of two opposite sides of $Q$, the left and right sides $L$ and $R$ in particular. This means that

$$
\sum_{i=1}^{n} t_{i} \geq \sum_{L} t_{i}+\sum_{R} t_{i}
$$

where the two sums correspond to those squares covering $L$ and $R$ respectively. Since these two sets of squares are disjoint, and each sum is at least $1, \sum_{i=1}^{n} t_{i} \geq 2$ is immediately implied. In particular, $V(n) \geq 2$ follows. We remark here that this simple argument for covering the perimeter would give only $U(n) \geq 3 / 2$ for the original covering problem with equilateral triangles.

The upper bound construction is as follows: Let $\varepsilon>0$ be arbitrary small, and set $\delta=\varepsilon$ for $n=4$, and $\delta=\frac{\varepsilon}{n-4}$ for $n \geq 5$. We cover $Q$ by a square $Q_{1}$ of side length $1-(n-3) \delta$ at the upper-left corner, a square $Q_{2}$ of side length $1-\delta$ at the lower-right corner, a square $Q_{3}$ of side length $(n-3) \delta$ at the lower-left corner, and $n-3$ squares of side lengths $\delta$ at the upper-right corner. We have a covering with $\sum_{i=1}^{n} t_{i}=2+(n-4) \delta \leq 2+\varepsilon$. Making $\varepsilon$ arbitrary small yields $V(n) \leq 2$. In conclusion, we have $V(n)=2$, as desired.

Finally, we would like to point out that the above lower bound for squares is a special case of the more general result of Soltan and É. Vásárhelyi $[1,6]$ : Let $C$ be a plane convex body that is covered by its homothetic copies $C_{1}, C_{2}, \ldots$ with positive coefficients $\lambda_{1}, \lambda_{2}, \ldots$, respectively, where each $\lambda_{i}<1$. Then $\sum_{i=1}^{\infty} \lambda_{i} \geq 2$. Nevertheless we have included our simple argument for the special case.

\section{References}

[1] P. Braß, W. Moser, and J. Pach, Research Problems in Discrete Geometry, Springer, New York, 2005.

[2] P. Erdős, Some of my favorite problems in number theory, combinatorics, and geometry, Resenhas, 2 (1995), 165-186.

[3] P. Erdős and R. Graham, On packing squares with equal squares, Journal of Combinatorial Theory Ser. A, 19 (1975), 119-123.

[4] P. Erdős and A. Soifer, Squares in a square, Geombinatorics, 4 (1995), 110-114.

[5] Z. Füredi, Covering a triangle with homothetic copies, in Discrete Geometry - in Honor of W. Kuperberg's 65th Birthday, A. Bezdek, ed., Marcel Dekker, 2003, 435-445.

[6] V. P. Soltan and É. Vásárhelyi, Covering a convex body by smaller homothetic copies, Geometriae Dedicata, 45 (1993), 101-113.

[7] Y. Zhang and Y. Fan, Packing and covering a unit equilateral triangle with equilateral triangles, Electronic Journal of Combinatorics, 12 (2005), \#R55. 